\title{
Detecting heterogeneity in single-cell RNA-Seq data by non- negative matrix factorization
}

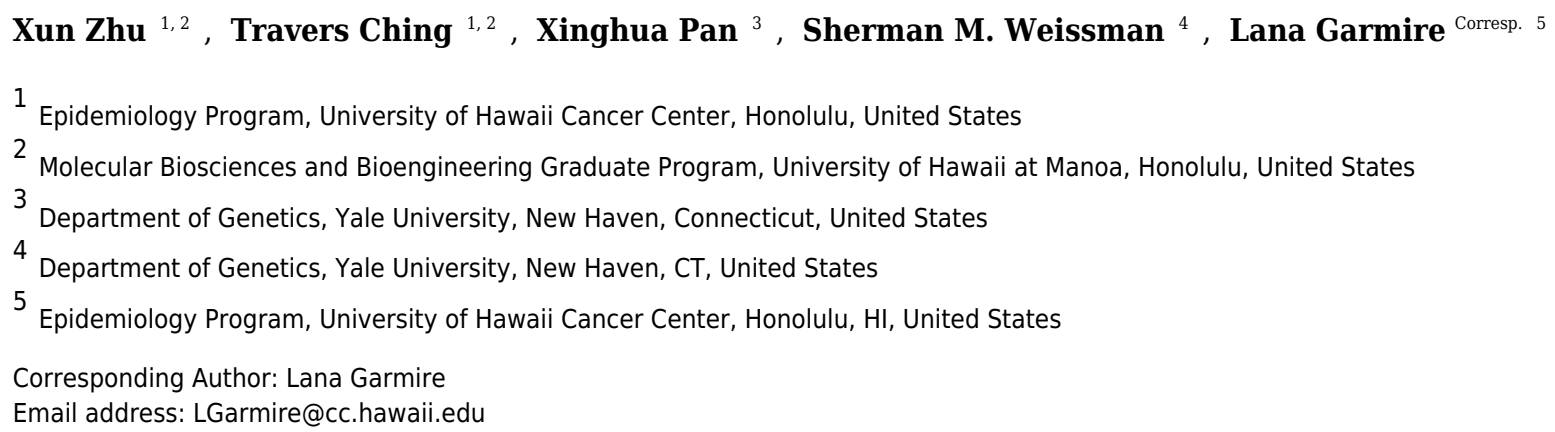

Single-cell RNA-Sequencing (scRNA-Seq) is a fast-evolving technology that enables the understanding of biological processes at an unprecedentedly high resolution. However, well-suited bioinformatics tools to analyze the data generated from this new technology are still lacking. Here we investigate the performance of non-negative matrix factorization (NMF) method to analyze a wide variety of scRNA-Seq datasets, ranging from mouse hematopoietic stem cells to human glioblastoma data. In comparison to other unsupervised clustering methods including K-means and hierarchical clustering, NMF has higher accuracy in separating similar groups in various datasets. We ranked genes by their importance scores (D-scores) in separating these groups, and discovered that NMF uniquely identifies genes expressed at intermediate levels as top-ranked genes. Finally, we show that in conjugation with the modularity detection method FEM, NMF reveals meaningful protein-protein interaction modules. In summary, we propose that NMF is a desirable method to analyze heterogeneous single-cell RNA-Seq data. The NMF based subpopulation detection package is available at: https://github.com/lanagarmire/NMFEM . 
1 Detecting heterogeneity in single-cell RNA-Seq data by non-negative matrix factorization

3

4 Xun Zhu ${ }^{1,2}$, Travers Ching ${ }^{1,2}$, Xinghua $\operatorname{Pan}^{3}$, Sherman Weissman ${ }^{3}$, Lana Garmire ${ }^{2, *}$

$5{ }^{1}$ Molecular Biosciences and Bioengineering Graduate Program, University of Hawaii at Manoa,

6 Honolulu, Hawaii, United States of America

$7 \quad 2$ Epidemiology Program, University of Hawaii Cancer Center, Honolulu, Hawaii, United States

8 of America

$9{ }^{3}$ Department of Genetics, Yale University, New Haven, Connecticut, United States of America

$11{ }^{*}$ Corresponding author.

12 Email address: 1garmire@cc.hawaii.edu 


\section{Abstract}

20 Single-cell RNA-Sequencing (scRNA-Seq) is a fast-evolving technology that enables the

21 understanding of biological processes at an unprecedentedly high resolution. However, well-

22 suited bioinformatics tools to analyze the data generated from this new technology are still

23 lacking. Here we investigate the performance of non-negative matrix factorization (NMF)

24 method to analyze a wide variety of scRNA-Seq datasets, ranging from mouse hematopoietic

25 stem cells to human glioblastoma data. In comparison to other unsupervised clustering methods

26 including K-means and hierarchical clustering, NMF has higher accuracy in separating similar

27 groups in various datasets. We ranked genes by their importance scores (D-scores) in separating

28 these groups, and discovered that NMF uniquely identifies genes expressed at intermediate levels

29 as top-ranked genes. Finally, we show that in conjugation with the modularity detection method

30 FEM, NMF reveals meaningful protein-protein interaction modules. In summary, we propose

31 that NMF is a desirable method to analyze heterogeneous single-cell RNA-Seq data. The NMF

32 based subpopulation detection package is available at: https://github.com/lanagarmire/NMFEM. 


\section{Introduction}

34 The advancement of technologies has enabled researchers to separate individual cells from bulk

35 and sequence their transcriptomes at the single cell level, known as single-cell RNA-Sequencing

36 (scRNA-Seq). This technology has reached an unprecedented fine resolution to reveal the

37 program of gene expression within cells (Kumar et al., 2014). It was used to detect heterogeneity

38 within the cell population, and it has greatly enhanced our understanding of the regulatory

39 programs involved in systems such as glioblastoma (Patel et al., 2014), neuronal cells (Usoskin

40 et al., 2014), or pluripotent stem cells (PSCs) (Kumar et al., 2014). It was also used to delineate

41 cell types and subpopulations in differentiating embryonic cells (Treutlein et al., 2014). Other

42 applications include uncovering multilineage priming processes involved in the initial

43 organogenesis (Brunskill et al., 2014), and substantiating the hypothesis of inter-blastomere

44 differences in 2- and 4-cell mouse embryos (Biase, Cao \& Zhong, 2014). Indeed, scRNA-Seq

45 has already made profound impacts on our understanding of the diversity, complexity, and

46 irregularity of biological activities in cells. It will continue to provide more transformative

47 insights in the near future (Pan, 2014; Poirion et al., 2016).

However, relative to the experimental technology, the bioinformatics tools to analyze scRNA-

49 Seq data are still lagging behind. Recently, various methods have been developed to detect

subpopulations (or sub-clusters) within a group of cells using scRNA-Seq data, including

51 scLVM (Buettner et al., 2015), BackSpin (Zeisel et al., 2015), PAGODA (Fan et al., 2016), and

52 SEURAT (Macosko et al., 2015). These new computational tools are evidence that

53 understanding scRNA-Seq heterogeneity is of paramount importance. Moreover, once the

54 subpopulations are identified, it is very crucial to find the gene expression signatures that are 
55 characteristic of each subpopulation (subclass), in order to reveal the subline biological

56 mechanisms.

57 Population-level RNA-Seq differential expression analysis, such as DESeq2 (Love, Huber \& 58 Anders, 2014) and edgeR (Robinson, McCarthy \& Smyth, 2010), are designed to compare pre-

59 labeled classes. However, it is questionable if they are desirable to identify subpopulations in

60 scRNA-Seq data. Recently, a couple of methods have been reported in the scRNA-Seq analysis

61 domain (Brennecke et al., 2013; McDavid et al., 2013; Kharchenko, Silberstein \& Scadden,

62 2014). For example, a statistical variance model based on gamma distribution was developed to

63 account for the high technical noise occurring in scRNA-seq experiments, such that genes with

64 high squared correlation of variations $\left(\mathrm{CV}^{2}\right)$ relative to mean expression were identified as

65 "significantly differentially expressed" between two conditions (Brennecke et al., 2013). Another

66 Bayesian approach was proposed for scRNA-Seq differential expression analysis, by utilizing a

67 probabilistic model of expression-magnitude distortions that were commonly observed in noisy

68 single-cell experiments (Kharchenko, Silberstein \& Scadden, 2014). This method later was used

69 for classification of sensory neurons using scRNA-Seq (Usoskin et al., 2014). On the other hand,

70 an R package, Monocle, was developed recently for single-cell lineage construction (Trapnell et

71 al., 2014).

72 Previously, NMF has been applied to other areas in computational biology, such as molecular

73 pattern discovery (Brunet et al., 2004), class comparison and prediction (Gao \& Church, 2005),

74 cross-platform and cross-species analysis (Tamayo et al., 2007), and identification of

75 subpopulations of cancer patients with mutations in similar network regions (ref). More recently,

76 NMF has been applied to gene expression profiling studies at the population level (Qi et al.,

77 2009). Compared to other methods, it showed multiple advantages, such as less sensitivity to $a$ 
78 priori selection of genes or initial conditions and the ability to detect context-dependent patterns

79 of gene expression (Rajapakse, Tan \& Rajapakse, 2004). Based on these properties, we

80 hypothesize that NMF is less prone to the influence of noise in the scRNA-Seq data, and thus it

81 can detect a group of genes that robustly differentiate single cells from different subpopulations.

82 In this report, we demonstrate the capabilities of NMF in scRNA-Seq data analysis in these

83 following aspects: (1) accurate clustering of single cells from different conditions in an

84 unsupervised manner; (2) detection of important genes associated with differences among

85 subclasses (subpopulations); (3) identification of protein-protein interaction modules around the

86 important genes, based on a modified implementation of Functional Epigenetic Modules (FEM)

87 (Jiao, Widschwendter \& Teschendorff, 2014). We organized the NMF workflow into a

88 streamlined modularity detection R package called NMFEM.

89 Materials \& Methods

90 Datasets

91 Five scRNA-Seq datasets are used in this study. For the first four datasets that don't have UMI,

92 normalized fragments per kilo-base per million reads (FPKM) is calculated and used as input to

93 the clustering methods. For the last dataset (iPSC) where UMI technique is adopted, we used the

94 author provided "molecule counts" as the input.

95 Mouse lung epithelial cells

96 scRNA-Seq data were retrieved from the original 201 samples of lung distal epithelial cells of

97 embryonic mouse (GSE52583) (Treutlein et al., 2014). The original dataset contains cells

98 collected from four time points: E14.5, E16.5, E18.5 and AT2. After filtering (the procedure is

99 detailed in the "Sample and Gene Filtering" section below), 201 single-cell samples and 4,594 
100 genes are used in downstream analysis. The closest pair of groups, which consists of 45 cells

101 from E14.5 and 27 cells from E16.5 was used for unsupervised clustering comparison.

\section{Mouse HSCs and MPP1s}

103 scRNA-Seq data were extracted from mouse hematopoietic stem cells (HSCs) and early

104 multipotent progenitors (MPP1s). The data were pre-processed into the format of a FPKM

105 expression profile (Table S1), which include 59 HSCs and 53 MPP1 single cells. After filtering,

10695 samples and 2,887 genes were used in downstream analysis.

\section{Glioblastoma}

108 scRNA-Seq data were retrieved from the original 875 samples of glioblastoma tumor cells in 5

109 patients (MGH26, MGH28, MGH29, MGH30, and MGH31), along with population and cell line

110 controls (GSE57872) (Patel et al., 2014). After filtering, 419 single-cell samples and 6,996 genes

111 were used in downstream analysis. The closest pair of groups, which consists of 80 cells from

112 patient MGH29 and 73 cells from MGH31 was used for unsupervised clustering comparison.

\section{Mouse bone marrow}

114 scRNA-Seq data were extracted from mouse macrophage DC progenitors (MDPs), common DC

115 progenitors (CDPs), and Pre-DCs (GSE60781) (Schlitzer et al., 2015). We used the FPKM table

116 provided by the authors. After filtering, 242 single-cell samples and 5,489 genes are used in

117 downstream analysis. The closest pair of groups, which consists of 59 MDPs and 89 CDPs was

118 used for the unsupervised clustering comparison.

\section{Human induced pluripotent stem cell lines}

120 scRNA-Seq data were extracted from induced pluripotent stem cell lines of three Yoruba

121 individuals. On each individual three technical replicates were performed. 5bp random sequence 
122 UMI were attached to the transcripts in order to avoid amplification bias. We used the molecule

123 counts provided by the authors. After filtering, 864 single-cell samples and 9,750 genes are used

124 in downstream analysis. The closest pair of groups, which consists of 288 cells from individual

125 NA19098 and 288 cells from individual NA19239 was used for the unsupervised clustering

126 comparison.

\section{Single-cell RNA-Seq analysis}

\section{Read alignment}

129 We downloaded the public datasets from NCBI The Gene Expression Omnibus (GEO) database

130 (Edgar, Domrachev \& Lash, 2002; Barrett et al., 2013), and retrieved the SRA files from The

131 Sequence Read Archive (SRA) (Leinonen et al., 2011). We used the fastq-dump tool from SRA

132 Toolkit to convert the SRA files into two pair-end FASTQ files. We applied FastQC for quality

133 control and TopHat2 (Kim et al., 2013) for alignment to the reference genomes. The ready-to-use

134 genome sequences and annotation files were downloaded from Illumina iGenomes page

135 (http://support.illumina.com/sequencing/sequencing_software/igenome.html). For human build

136 hg19 was used, and for mouse genome build mm10 was used (Karolchik et al., 2014).

\section{Read Counting}

138 We used featureCounts (Liao, Smyth \& Shi, 2014) to map and count the aligned BAM files to

139 the RefSeq transcriptomes from the pre-built packages on Illumina iGenome website above. We

140 used the options to count fragments instead of reads; paired-end distance was checked by

141 featureCounts when assigning fragments to meta-features or features. We only took into account

142 of fragments that have both ends aligned successfully and discarded chimeric fragments. 
143 Fragments mapped to multiple locations were counted. The command is "featureCounts -

144 pPBCM --primary -T 6 -a $<$ gtf_file $>$-o < output_file $><$ bam_file $>$ ".

\section{Normalization of Counts}

146 We used reads per kilo base per million (FPKM) to represent the gene expression level, where

147 the length of each gene was calculated by UCSC RefSeq annotation table, by concatenating all

148 the exons. We normalized the data using DESeq2.

\section{Sample and Gene Filtering}

150 Samples expressing housekeeping genes with geometric average FPKM lower than 4 were

151 deemed abnormal and removed. Genes that were not expressed at all in over $70 \%$ of the cells

152 were removed.

\section{Closest Pair Identification}

154 The closest pair of groups among the samples is defined as the pair that has the smallest distance

155 between the two groups' centroids, on the 2-dimentional correlation t-SNE plot. The correlation $156 \mathrm{t}-\mathrm{SNE}$ is the t-SNE method (Van der Maaten \& Hinton, 2008) performed on the correlation

157 distance between two samples $\mathrm{x}$ and $\mathrm{y}$ :

$$
d_{c}(x, y)=1-\operatorname{cor}(x, y)
$$

\section{Non-negative Matrix Factorization (NMF)}

160 We used the R-package implementation of NMF (Gaujoux \& Seoighe, 2010) to perform NMF

161 analysis. NMF is mathematically approximated by: $A \approx W H$, where $A$ ( $n$ by $m$ ) is the matrix

162 representing the scRNA-Seq profile in this report, $W$ is a slim weight matrix ( $n$ by $k$, where

$163 n \gg k$ ), $H$ is a wide matrix ( $k$ by $m$, where $m \gg k$ ), and all three of them are non-negative 
164 (Brunet et al., 2004). The column vectors in $W$ are called meta-genes, which are higher-level

165 abstraction of the original gene expression pattern. For gene $i$, the loadings are the $k$ values in $W$

166 at row $i$. We used the method "brunet" to solve the approximation of $A$, which employs the

167 multiplicative iterative algorithm described by the following rules:

168

$$
H_{a u} \leftarrow H_{a u} \frac{\sum_{i} \frac{W_{i a} V_{i u}}{(W H)_{i u}}}{\sum_{k} W_{k a}}
$$

169

$$
W_{i a} \leftarrow W_{i a} \frac{\sum_{u} \frac{H_{a u} A_{i u}}{(W H)_{i u}}}{\sum_{v} H_{a v}} .
$$

170 The initialization of $H_{a u}$ and $W_{i a}$ was generated as random seed matrices drawn from a uniform

171 distribution within the same range as the entries in the matrix $A$. Since the starting matrices were

172 randomized, we conducted an average of 30 simulations for each NMF run to obtain the

173 consensus clustering results. We used Kullback-Leibler divergence (KL-divergence) as the

174 distance function, as it has significantly better performance theorized in Yang et al. (Yang et al.,

175 2011). The clustering results of all possible $k$ 's (usually ranging from 2 to 5 , as higher $k$ values

176 require exponentially more time to run) were listed and $k$ was chosen when the best cophenetic

177 correlation coefficient is achieved, as proposed in Brunet et al. 2004 (Brunet et al., 2004).

\section{D-score}

179 To rank the importance of genes using the two matrices factorized by NMF, we define D-score

180 as follow: The D-score for gene $i$ is defined as:

181

$$
D_{i}=\left|\lambda W_{i, 1}-(1-\lambda) W_{i, 2}\right|
$$


182 The balancing factor $\lambda$ is determined by the rule that a gene uniformly expressed across all

183 samples should have D-score 0 . This score is a slight modification of the gene ranking method

184 using discriminant NMF proposed earlier (Jia et al., 2015).

\section{Other packages used for detecting significant or important genes}

186 DE methods for bulk-level RNA-Seq: we used two most popular bulk-level RNA-Seq methods:

187 DESeq2 and edgeR, to compare on the results of DE genes.

188 DE methods for scRNA-Seq: three methods were investigated, with default settings of the

189 packages. (1) Monocle: this is a versatile method (V 1.0.0) that performs differential expression

190 analysis between cell types or states, moreover places cells in order according to their

191 progression through processes such as cell differentiation (Trapnell et al., 2014). (2) SCDE: this

192 package (V 1.2.1) implemented in $\mathrm{R}$ is based on Bayesian method, where the individual genes

193 were modeled explicitly as a mixture of the dropout and amplification events by the Poisson

194 model and negative binomial model (Kharchenko, Silberstein \& Scadden, 2014). (3) MAST: this

195 method (V 1.0.1) implemented in R was originally used to detected DE genes in qPCR results of

196 single cells. We selected the 500 genes with the lowest likelihood ratio test $p$-value using Hurdle

197 Model provided by the package, as recommended by the authors (McDavid et al., 2013).

198 Additionally, we experimented if introducing t-SNE, a dimension reduction method that was

199 recently successfully applied to scRNA-Seq, would improve the results of NMF. We used the

$200 \mathrm{C}++$ accelerated R-package Rtsne (V 0.10), based on the original C++ implementation (van der

201 Maaten, 2013). 


\section{Module detection package}

203 We used Functional Epigenetic Modules (FEM) R package (Jiao, Widschwendter \&

204 Teschendorff, 2014) for module detection. FEM utilizes an expansion algorithm based on the z-

205 score of the expression level, by using a list of seed genes as the starting point. It selects the top

206 modules based on $p$-values calculated by a Monte Carlo method.

207 We modified the source code of the FEM package and changed the process of the seed gene

208 selection. Instead of selecting the seed genes based on the z-score of the expression level, we

209 directly plugged in a list of genes as the seed genes, which were generated from each of the

210 compared method for important gene detection.

\section{Measuring the performance of unsupervised clustering methods}

212 Pair-wise Rand measure for clustering between the test and the reference is defined by

$$
R=\frac{T P+T N}{T P+F P+F N+T N}
$$

214 in which the four quantities $T P, F P, F N$, and $T N$ are cardinals of the four sets of pairs. $T / F$

215 means true/false based on the reference, and $P / N$ means positive/negative results from the test.

216 Specifically, a positive result $(P)$ refers to a pair of samples clustered in the same group by the

217 tested method; a true positive $(T P)$ or true negative $(T N)$ result represents the case where the

218 agreements between the test and the reference clustering is reached (Rand, 1971).

\section{Modularity detection and pathway Analysis}

220 We used Functional Epigenetic Modules (FEM) package (Jiao, Widschwendter \& Teschendorff, 221 2014) implemented in R for module detection. FEM utilizes SpinGlass algorithm (Reichardt \& 
222 Bornholdt, 2006) based on the z-score of the expression level, by using a list of seed genes as the

223 starting points. It selects the top modules based on $p$-values calculated from a Monte Carlo

224 method. We modified the source code of the package to allow seed genes generated from other

225 methods (NMF, DESeq2, edgeR, SCDE, MAST and Monocle) that detect significant or

226 important genes. In each case, we used top 500 most important genes as the seeds for FEM. We

227 next compared biological meanings of the resulting modules by Gene Ontology (GO) or Kyoto

228 Encyclopedia of Genes and Genomes (KEGG) pathway enrichment analysis, implemented as

229 DAVID Web Service in R (Huang, Sherman \& Lempicki, 2008, 2009).

\section{Data and code availability}

231 The Glioblastoma, mouse lung distal epithelial, mouse bone marrow, and human induced

232 pluripotent stem cell lines datasets are downloaded from Gene Expression Omnibus (GEO)

233 repository with accession codes GSE57872, GSE52583, GSE60781, and GSE77288,

234 respectively. The code used for the package NMFEM can be found at

235 https://github.com/lanagarmire/NMFEM, and https://github.com/lanagarmire/NMFEM_extra.

\section{Results}

237 The workflow for a typical single-cell analysis using NMF is shown in Fig. 1. Briefly, the 238 pipeline can take raw reads from FASTQ files, align and count them to the transcriptome; or it

239 can use raw count data directly as the input matrix. The input data matrix is then subject to

240 quality control and normalization steps. NMF then operates on the normalized matrix, clusters

241 the samples into subpopulations (or subclasses) and enlists the feature genes that separate the

242 subpopulations. In order to display the insightful biological modules and hotspots in the 
243 interactome, the feature genes are then used as seeds for a functional modularity detection

244 algorithm FEM (Jiao, Widschwendter \& Teschendorff, 2014).

245 NMF accurately clusters scRNA-Seq data among similar

\section{6 populations}

247 We first assess NMF's accuracy in unsupervised clustering compared to two other commonly

248 used methods: K-means and hierarchical clustering (Hclust) algorithms. For generality, different

249 distance metric and linkage variations of Hcust clustering were explored, including the

250 combinations of Euclidean Distance \& Complete Linkage (Euclidean +Complete), Euclidean

251 Distance \& Ward Linkage (Euclidean +Ward), and Correlation Metric \& Complete Linkage

252 (Correlation+Complete). To draw unbiased conclusions, we tested these clustering methods on

253 five datasets, including a mouse embryonic lung epithelial dataset (Treutlein et al., 2014), a

254 mouse hematopoietic stem cell (HSC) and multipotent progenitor (MPP) cell dataset (Table S1),

255 a human glioblastoma dataset (Patel et al., 2014), a mouse bone marrow dendritic cells dataset

256 (Schlitzer et al., 2015), and lastly a human induced pluripotent stem cell (iPSC) dataset with

257 unique molecular identifier (UMI) counts (Tung et al., 2016). A good clustering method should

258 be able to separate groups that are most similar, therefore we selected the closet two groups in

259 each dataset (Methods).

260 As shown in Fig. 2, NMF consistently achieves the highest performance among all methods

261 compared, as measured by the Rand Index measure (Methods). Indeed, despite the high level of

262 similarity between each pair of groups, NMF achieves an average Rand measure of 0.88 among

263 the five datasets. In particular, it has an impressive Rand measure of 0.95 in the mouse

264 embryonic lung epithelial dataset (Fig. 2A) and almost 1.00 in the UMI iPSC dataset (Fig. 2E). 
265 In contrast, K-means and the three variations of hierarchical clustering (Euclidean + Complete,

266 Euclidean + Ward, and Correlation + Complete) all have significantly (p-value $<0.05)$ lower

267 averaged Rand measures of $0.66,0.52,0.66$, and 0.52 , respectively (Fig. 2). Most of the wrongly

268 clustered samples by K-means are located between the two clusters in the Euclidean space (Fig.

269 S1 A). And hierarchical clustering methods can be heavily affected by individual outliers (Fig.

270 S1 B-D).

271 t-SNE is a commonly used dimension reduction method that exaggerates the differences between

272 populations (Van der Maaten \& Hinton, 2008), and was previously used in combination with

273 other clustering methods on scRNA-Seq data(Van der Maaten \& Hinton, 2008; Bushati et al.,

274 2011; Junker et al., 2014). Therefore, we investigated the effect of t-SNE preprocessing on NMF

275 and other compared methods next. Interestingly, NMF performs significantly worse after t-SNE

276 preprocessing (Fig. 2). We speculate that the more significant decrease in accuracy by NMF is

277 attributed to the fact that NMF is not a distance-based method, and the reduction of features after

278 t-SNE interferes with NMF's ability to conduct component decomposition more drastically than

279 other methods.

280 NMF discovers biologically meaningful genes to separate subpopulations

281 One major advantage of NMF over many other unsupervised clustering methods such as K-

282 means, is its ability to simultaneously identify genes that are characteristic to each

283 subpopulations. We use the discriminative metric called D-score, based the previous rank-2

284 discriminant NMF implementation (Jia et al., 2015). Briefly, D-score is a non-negative number,

285 and the more positive a gene's D-score is, the more unique this gene is expressed in a

286 subpopulation (Methods). We ranked the top genes in NMF by D-score in the descending order,

287 and sought prior literature reports to validate the utilities of NMF empirically. 
288 For the top ranked NMF genes differentiating E14.5 and E16.5 (Treutlein et al., 2014), we found 289 biological evidence to support their functions. For example, Mal, T-Cell Differentiation Protein 2902 (Mal2), ranked $2^{\text {nd }}$ by NMF, was noted to be involved in the dynamic circuit of early lung 291 development (Ye, Liu \& Wu, 2012). Another gene Surfactant Associated 2 (Sfta2), ranked $4^{\text {th }}$ by 292 NMF, was recently identified as an expression QTL (eQTL) gene during early lung development 293 (George et al., 2016). Additionally, we examined top genes differentiating the subsets of AT1 vs. 294 AT2 samples as reported originally (Treutlein et al., 2014). Impressively, the four genes Hopx, 295 Ager, Egfl6, and Sftpc which were marker genes reported in the original report, have ranks of $1^{\text {st }}$, $29612^{\text {th }}, 23^{\text {rd }}$ and $27^{\text {th }}$ among the top genes according to NMF.

297 Similarly, in the HSC vs. MPP1 dataset, many of the top-ranked genes were previously noted as 298 either differentially expressed between the two types of cells or characteristic to one of the two 299 types. For example, Cysteine Rich Protein 1 (Crip1), ranked $2^{\text {nd }}$ by NMF D-score, is found to be 300 expressed lower in Hdac3 knock-out cells undergoing HSC and MPP differentiation (Summers et 301 al., 2013). Regulator of G-Protein Signaling 2 (Rgs2), ranked $3^{\text {rd }}$ by NMF, was noted to be 302 differentially expressed in HSCs (Phillips et al., 2000; Li \& Akashi, 2003; Park et al., 2003). 303 SKI-Like Proto-Oncogene (Skil), ranked $8^{\text {th }}$ by NMF, was shown to play important roles in 304 hematopoietic development (Pearson-White et al., 1995).

305 For the MGH29 and MGH31 glioblastoma patient data (Patel et al., 2014), although we could 306 not obtain definite clinical subtypes based on the original paper, we did observe that many top 307 genes identified had previously been related to various types of cancers. For example,

308 Chromosome 8 Open Reading Frame 4 (C8orf4), ranked 9th by NMF, was identified as "Thyroid

309 cancer 1 (TC1)"(Panebianco et al., 2015; Zhang et al., 2015; Zhu et al., 2015; Huang et al., 310 2016). Epidermal Growth Factor Receptor (EGFR), a gene ranked $31^{\text {st }}$ by NMF, has elevated 
311 expression from MGH29 to MGH31. EGFR is a well-known growth factor receptor noted for its

312 critical role on cell division control. Moreover, three top genes EZR (ranked 12 ${ }^{\text {th }}$ ), TUBB

313 (ranked $27^{\text {th }}$ ), and $\mathrm{RDX}$ (ranked $36^{\text {th }}$ ), were shown to be involved in organization and regulation

314 of morphological characteristics of breast cancer cells including cell shape and membrane to

315 membrane docking (Kopp et al., 2016).

\section{Comparison of important genes from NMF and other methods}

317 To further understand the patterns recognized by NMF, we compared the top ranked genes

318 identified by NMF with the those by other recent scRNA-Seq analysis methods, including

319 Monocle (Trapnell et al., 2014), MAST (McDavid et al., 2013) as well as SCDE (Kharchenko,

320 Silberstein \& Scadden, 2014) (Treutlein et al., 2014). In addition, we included DESeq2 and

321 edgeR, two differential expression (DE) methods commonly used in bulk RNA-Seq analysis.

322 We compared the different methods using MA plots to analyze the mouse embryonic lung

323 epithelial cells dataset (E14.5 and E16.5, Fig. 3). For fair-comparison, we highlighted the top 500

324 genes identified by each method in blue. Since the global gene expression levels are reduced

325 from E14.5 to E16.5, all methods detect mainly down-regulated genes. The specific genes

326 identified by these methods however vary greatly (Fig. 3, Fig. S2 and Fig. S3), but NMF is most

327 different from other methods, based on its unique mathematical formulation. Unlike other

328 methods that are prone to lowly expressed genes, NMF tends to select genes that have

329 intermediate expression levels (Fig. S3). Indeed, 90\% of selected genes have log FPKM between

3302.32 and 4.66. Other methods, on the other hand, select more genes with lower expression levels.

331 Specifically, SCDE, MAST, DESeq2, edgeR and Monocle have $56 \%, 45 \%, 31 \%, 27 \%$ and $18 \%$

332 genes below FPKM=1, respectively. The same pattern has been observed in the HSC vs. MPP,

333 glioblastoma, and bone marrow dataset (Fig. S4 A-C). Lowly expressed genes usually have 
334 relatively higher levels of noise (Brennecke et al., 2013), evident by comparing the CV2 levels

335 between genes selected by different methods (Fig. S5), and their apparent differential expression

336 may be less reliable.

337 We further tested whether such intermediately expressed genes identified by NMF are robust and

338 unlikely a result of random sampling. We performed additional runs with each run deliberately

339 dropping one sample from the dataset. The results show that the top 500 genes have over $95 \%$

340 probability to re-appear in this top 500 list, compared to less than $2 \%$ probability for any other

341 non-top genes (Fig. S6). The reason that NMF tends to avoid lowly expressed genes is that D-

342 score considers absolute expression level of a gene, rather than the relative expression level (see

343 Methods). The lower its expression level, the less likely a gene is selected as a feature gene by

344 NMF. On the other hand, very highly expressed genes are more likely to be expressed across all

345 cells. They do not generate large differences in their meta-gene loadings, and will not achieve

346 high D-score as well.

347 Modularity detection with NMF based framework

348 We next asked if the important genes detected by NMF convey unique and meaningful biological

349 functions. Previously, a community detection method based on SpinGlass algorithm named FEM

350 has been reported to detect functional modules on protein-protein interaction (PPI) networks

351 (Jiao, Widschwendter \& Teschendorff, 2014). Inspired by this approach, we used the 500 top

352 genes selected by NMF as seed genes, and ran vertex-initialized SpinGlass models on the PPI

353 network. Modified from FEM, here each vertex (gene) is weighted by t-statistics measuring the

354 expression level (rather than DNA methylation) changes. In order to assess the robustness of the

355 method, we performed random permutations on the data and calculated the $p$-value for each

356 module. The top four modules for mouse embryonic lung epithelial cells dataset are shown in 
357 Fig. 4. We conducted the same procedure on HSC vs. MPP1, glioblastoma, and bone marrow 358 datasets (Fig. S4 D-F).

359 Consistent with the down-regulating trend of all genes from E14.5 to E16.5 (Fig. 3), these top

360 four modules exhibit strong down-regulation as well. Five hub genes are identified: Rad21, Atf3,

361 Polr2j, Tecr and Rpl37, among which Tecr and Rpl37 are in the same module. These modules

362 are involved in chromosome structural maintenance (seed Rad21), transcription activation (seed

363 Atf3), RNA polymerase (seed Polr2j), and ribosome component and various enzyme coding

364 genes (Tecr and Rpl37). The fact that the top modules are all related to transcription and

365 translation is interesting, as it explains the observed global gene expression down-regulation

366 observed earlier (Fig. 3).

\section{Discussion \& Conclusions}

368 Due to the high level of noise in scRNA-Seq data (Brennecke et al., 2013) and its unique

369 challenges to elucidate the subtle relationships of a seemingly similar single cells in a population,

370 the traditional DE approaches that rely on known labels and strong expression differences of

371 single genes may be limited. Meanwhile, NMF with the ability to capture the general expression

372 pattern changes may be very suitable for scRNA-Seq subpopulation identification. Previous

373 applications of NMF to fields such as face reorganization (Rajapakse, Tan \& Rajapakse, 2004),

374 image compression (Yuan \& Oja, 2005; Monga \& Mihçak, 2007) and sound decomposition

375 (Smaragdis, 2004), have been proven successful. Here we propose to utilize NMF as a suitable

376 method for scRNA-Seq analysis.

377 Specifically, we have demonstrated that in multiple datasets and multiple scRNA-Seq protocols,

378 NMF performed significantly better than other popular clustering methods including K-means 
379 and hierarchical clustering. Moreover, since NMF is an unsupervised method, it is not affected

380 by preconceived cell type labeling. Its higher accuracy and additional ability to extract feature

381 genes may allow it to serve as the direct substitute to other commonly used unsupervised

382 methods. It should be noted however, that this benefit of NMF diminishes when the classes are

383 drastically different. Also, the fact that it has the tendency to select more highly expressed and

384 correlated genes implies that it might miss biologically important genes (such as transcription

385 factors) that have low expression levels (Tian et al., 2011).

386 Another caveat of our secondary data analysis on public data set, is that there are batch effects

387 that confound the patterns recognized by NMF (or any other subpopulation detection methods).

388 In these experiments, groups of samples are sequenced in different runs, thus technical variation

389 may confound the biological variation (Hicks, Teng \& Irizarry, 2015). We calculated the

390 confoundedness of the three datasets (mouse embryonic lung epithelial, glioblastoma and bone

391 marrow datasets) where experimental information is available, and found that confounding

392 accounts $92.8 \%, 98.9 \%$ and $100 \%$, respectively. However, since NMF is able to identify some

393 previously validated biological results, it is reasonable to believe that NMF still has the power to

394 detect biological factors. It is nonetheless paramount to design experiments well so that such

395 confounding effects can be minimized in the future scRNA-Seq studies.

396 Moreover, the best combinatory approaches for scRNA-Seq subpopulation identification will

397 continue to be an interesting area of research. For example, a few normalization methods have

398 been proposed for RNA-Seq experiments lately, including RUV (Risso et al., 2014) and GRM

399 (Ding et al., 2015). These normalization methods may have additional impact on the results of

400 clustering. More rigorous source of variation (SOV) analysis to identify the best combinations of

401 analysis steps will be an interesting follow-up project. 
402 In summary, we have demonstrated that NMF is a method capable of accomplishing various

403 tasks in scRNA-Seq data analysis, from reclassifying populations of single cells to revealing

404 meaningful genes and modules of biological significance. We expect the new workflow

405 proposed here will have valuable applications in the field of scRNA-Seq bioinformatics analysis.

\section{Acknowledgement}

407 We would like to thank our colleagues Kumardeep Chaudhary and Olivier Poirion for reading 408 the manuscript and providing valuable suggestions.

\section{References}

410 Barrett T., Wilhite SE., Ledoux P., Evangelista C., Kim IF., Tomashevsky M., Marshall KA., 411 Phillippy KH., Sherman PM., Holko M., Yefanov A., Lee H., Zhang N., Robertson CL., 412 Serova N., Davis S., Soboleva A. 2013. NCBI GEO: archive for functional genomics data sets—update. Nucleic Acids Research 41:D991-D995. DOI: 10.1093/nar/gks1193.

414 Biase F., Cao X., Zhong S. 2014. Cell fate inclination within 2-cell and 4-cell mouse embryos 415 revealed by single-cell RNA sequencing. Genome research:gr-177725.

416 Brennecke P., Anders S., Kim JK., Kołodziejczyk AA., Zhang X., Proserpio V., Baying B.,

417 Benes V., Teichmann SA., Marioni JC. 2013. Accounting for technical noise in single-cell 418 RNA-seq experiments. Nature methods.

419 Brunet J-P., Tamayo P., Golub TR., Mesirov JP. 2004. Metagenes and molecular pattern 420 discovery using matrix factorization. Proceedings of the National Academy of Sciences $421 \quad 101: 4164-4169$. 
422 Brunskill EW., Park J-S., Chung E., Chen F., Magella B., Potter SS. 2014. Single cell dissection 423 of early kidney development: multilineage priming. Development 141:3093-3101.

424 Buettner F., Natarajan KN., Casale FP., Proserpio V., Scialdone A., Theis FJ., Teichmann SA., 425 Marioni JC., Stegle O. 2015. Computational analysis of cell-to-cell heterogeneity in single426 cell RNA-sequencing data reveals hidden subpopulations of cells. Nature biotechnology $427 \quad 33: 155-160$.

428

431

432

433

434

435

436

437

438

Bushati N., Smith J., Briscoe J., Watkins C. 2011. An intuitive graphical visualization technique for the interrogation of transcriptome data. Nucleic acids research 39:7380-9. DOI: 10.1093/nar/gkr462.

Ding B., Zheng L., Zhu Y., Li N., Jia H., Ai R., Wildberg A., Wang W. 2015. Normalization and noise reduction for single cell RNA-seq experiments. Bioinformatics:btv 122.

Edgar R., Domrachev M., Lash AE. 2002. Gene Expression Omnibus: NCBI gene expression and hybridization array data repository. Nucleic Acids Research 30:207-210. DOI: 10.1093/nar/30.1.207.

Fan J-BJJ-BJ., Salathia N., Liu R., Kaeser GE., Yung YC., Herman JL., Kaper F., Fan J-BJJ-BJ., Zhang K., Chun J., others. 2016. Characterizing transcriptional heterogeneity through pathway and gene set overdispersion analysis. Nature methods.

Gao Y., Church G. 2005. Improving molecular cancer class discovery through sparse nonnegative matrix factorization. Bioinformatics 21:3970-3975.

Gaujoux R., Seoighe C. 2010. A flexible R package for nonnegative matrix factorization. BMC bioinformatics 11:367. 
443 George L., Irmler M., Mitra A., Thimraj TA., Beckers J., Upadhyay S., Schulz H., Leikauf GD.,

444 Ganguly K. 2016. Mouse Lung Developmental Gene Expression Profiling Study Identifies

445 Novel Candidate Genes For Pulmonary Function. In: B58. BIG AND BIGGER (DATA):

446

447 OMICS AND BIOMARKERS OF COPD AND OTHER CHRONIC LUNG DISEASES. Am

Hicks SC., Teng M., Irizarry RA. 2015. On the widespread and critical impact of systematic bias and batch effects in single-cell RNA-Seq data. bioRxiv:25528.

Huang M., Zhong Z., Lv M., Shu J., Tian Q., Chen J. 2016. Comprehensive analysis of differentially expressed profiles of lncRNAs and circRNAs with associated co-expression

Huang DW., Sherman BT., Lempicki RA. 2008. Systematic and integrative analysis of large gene lists using DAVID bioinformatics resources. Nature protocols 4:44-57.

Huang DW., Sherman BT., Lempicki RA. 2009. Bioinformatics enrichment tools: paths toward 456 the comprehensive functional analysis of large gene lists. Nucleic acids research 37:1-13.

Jia Z., Zhang X., Guan N., Bo X., Barnes MR., Luo Z. 2015. Gene Ranking of RNA-Seq Data via Discriminant Non-Negative Matrix Factorization. PloS one 10:e0137782.

Jiao Y., Widschwendter M., Teschendorff AE. 2014. A systems-level integrative framework for 460 genome-wide DNA methylation and gene expression data identifies differential gene 461 expression modules under epigenetic control. Bioinformatics:btu316.

462 Junker JP., Noël ES., Guryev V., Peterson KA., Shah G., Huisken J., McMahon AP., Berezikov 463 E., Bakkers J., van Oudenaarden A. 2014. Genome-wide RNA Tomography in the 
465 Karolchik D., Barber GP., Casper J., Clawson H., Cline MS., Diekhans M., Dreszer TR., Fujita

466 PA., Guruvadoo L., Haeussler M. 2014. The UCSC genome browser database: 2014 update. $467 \quad$ Nucleic acids research 42:D764-D770.

468

469

470

471

472

Kharchenko P V., Silberstein L., Scadden DT. 2014. Bayesian approach to single-cell differential expression analysis. Nature methods 11:740-742.

Kim D., Pertea G., Trapnell C., Pimentel H., Kelley R., Salzberg SL. 2013. TopHat2: accurate alignment of transcriptomes in the presence of insertions, deletions and gene fusions. Genome Biol 14:R36.

Kopp S., Slumstrup L., Corydon TJ., Sahana J., Aleshcheva G., Islam T., Magnusson NE., Wehland M., Bauer J., Infanger M. 2016. Identifications of novel mechanisms in breast cancer cells involving duct-like multicellular spheroid formation after exposure to the Random Positioning Machine. Scientific reports 6.

Kumar RM., Cahan P., Shalek AK., Satija R., DaleyKeyser AJ., Li H., Zhang J., Pardee K., Gennert D., Trombetta JJ., Ferrante TC., Regev A., Daley GQ., Collins JJ. 2014. Deconstructing transcriptional heterogeneity in pluripotent stem cells. Nature 516:56-61. DOI: $10.1038 /$ nature 13920 .

Leinonen R., Sugawara H., Shumway M., Collaboration on behalf of the INSD. 2011. The Sequence Read Archive. Nucleic Acids Research 39:D19-D21. DOI: 10.1093/nar/gkq1019.

Li L., Akashi K. 2003. Unraveling the molecular components and genetic blueprints of stem cells. Biotechniques 35:1233-1239. 
485 Liao Y., Smyth GK., Shi W. 2014. featureCounts: an efficient general purpose program for 486 assigning sequence reads to genomic features. Bioinformatics 30:923-930.

487 Love MI., Huber W., Anders S. 2014. Moderated estimation of fold change and dispersion for $488 \quad$ RNA-Seq data with DESeq2. bioRxiv.

van der Maaten L. 2013. Barnes-hut-sne. arXiv preprint arXiv:1301.3342.

Van der Maaten L., Hinton G. 2008. Visualizing data using t-SNE. Journal of Machine Learning Research 9:85.

492

494

Macosko EZ., Basu A., Satija R., Nemesh J., Shekhar K., Goldman M., Tirosh I., Bialas AR., Kamitaki N., Martersteck EM. 2015. Highly parallel genome-wide expression profiling of individual cells using nanoliter droplets. Cell 161:1202-1214.

McDavid A., Finak G., Chattopadyay PK., Dominguez M., Lamoreaux L., Ma SS., Roederer M., Gottardo R. 2013. Data exploration, quality control and testing in single-cell qPCR-based gene expression experiments. Bioinformatics (Oxford, England) 29:461-7. DOI: 10.1093/bioinformatics/bts714.

Monga V., Mihçak MK. 2007. Robust and secure image hashing via non-negative matrix factorizations. Information Forensics and Security, IEEE Transactions on 2:376-390.

Pan X. 2014. Single Cell Analysis: From Technology to Biology and Medicine. Single cell biology 3:106. DOI: 10.4172/2168-9431.1000106.

Panebianco F., Mazzanti C., Tomei S., Aretini P., Franceschi S., Lessi F., Di Coscio G., Bevilacqua G., Marchetti I. 2015. The combination of four molecular markers improves thyroid cancer cytologic diagnosis and patient management. BMC cancer 15:1. 
506 Park I., Qian D., Kiel M., Becker MW., Pihalja M., Weissman IL., Morrison SJ., Clarke MF.

507 2003. Bmi-1 is required for maintenance of adult self-renewing haematopoietic stem cells.

$508 \quad$ Nature 423:302-305.

509 Patel AP., Tirosh I., Trombetta JJ., Shalek AK., Gillespie SM., Wakimoto H., Cahill DP., Nahed

510 B V., Curry WT., Martuza RL., Louis DN., Rozenblatt-Rosen O., Suvà ML., Regev A.,

511 Bernstein BE. 2014. Single-cell RNA-seq highlights intratumoral heterogeneity in primary

512 glioblastoma. Science 344:1396-1401. DOI: 10.1126/science.1254257.

513 Pearson-White S., Deacon D., Crittenden R., Brady G., Iscove N., Quesenberry PJ. 1995. The

514 ski/sno protooncogene family in hematopoietic development. Blood 86:2146-2155.

515 Phillips RL., Ernst RE., Brunk B., Ivanova N., Mahan MA., Deanehan JK., Moore KA., Overton

516 GC., Lemischka IR. 2000. The genetic program of hematopoietic stem cells. Science

$517 \quad 288: 1635-1640$.

518 Poirion OB., Zhu X., Ching T., Garmire L. 2016. Single-Cell Transcriptomics Bioinformatics 519 and Computational Challenges . Frontiers in Genetics 7:163.

520 Qi Q., Zhao Y., Li M., Simon R. 2009. Non-negative matrix factorization of gene expression 521 profiles: a plug-in for BRB-ArrayTools. Bioinformatics 25:545-547.

522 Rajapakse M., Tan J., Rajapakse J. 2004. Color channel encoding with NMF for face

523 recognition. In: Image Processing, 2004. ICIP'04. 2004 International Conference on. IEEE, $524 \quad 2007-2010$.

525 Rand WM. 1971. Objective criteria for the evaluation of clustering methods. Journal of the $526 \quad$ American Statistical association 66:846-850. 
527 Reichardt J., Bornholdt S. 2006. Statistical mechanics of community detection. Physical Review $528 \quad E 74: 16110$.

529 Risso D., Ngai J., Speed TP., Dudoit S. 2014. Normalization of RNA-seq data using factor $530 \quad$ analysis of control genes or samples. Nature biotechnology 32:896-902.

531 Robinson MD., McCarthy DJ., Smyth GK. 2010. edgeR: a Bioconductor package for differential 532 expression analysis of digital gene expression data. Bioinformatics 26:139-140.

533 Schlitzer A., Sivakamasundari V., Chen J., Sumatoh HR Bin., Schreuder J., Lum J., Malleret B., 534 Zhang S., Larbi A., Zolezzi F. 2015. Identification of cDC1-and cDC2-committed DC 535 progenitors reveals early lineage priming at the common DC progenitor stage in the bone 536 marrow. Nature immunology 16:718-728.

537

538

539

540

541

542

543

544

545

546 547

Smaragdis P. 2004. Non-negative matrix factor deconvolution; extraction of multiple sound sources from monophonic inputs. In: Independent Component Analysis and Blind Signal Separation. Springer, 494-499.

Summers AR., Fischer MA., Stengel KR., Zhao Y., Kaiser JF., Wells CE., Hunt A., Bhaskara S., Luzwick JW., Sampathi S. 2013. HDAC3 is essential for DNA replication in hematopoietic progenitor cells. The Journal of clinical investigation 123:3112-3123.

Tamayo P., Scanfeld D., Ebert BL., Gillette MA., Roberts CWM., Mesirov JP. 2007. Metagene projection for cross-platform, cross-species characterization of global transcriptional states. Proceedings of the National Academy of Sciences 104:5959-5964.

Tian H., Biehs B., Warming S., Leong KG., Rangell L., Klein OD., de Sauvage FJ. 2011. A reserve stem cell population in small intestine renders Lgr5-positive cells dispensable. 
Nature 478:255-259.

549 Trapnell C., Cacchiarelli D., Grimsby J., Pokharel P., Li S., Morse M., Lennon NJ., Livak KJ.,

550 Mikkelsen TS., Rinn JL. 2014. Pseudo-temporal ordering of individual cells reveals

551 dynamics and regulators of cell fate decisions. Nature biotechnology 32:381.

552 Treutlein B., Brownfield DG., Wu AR., Neff NF., Mantalas GL., Espinoza FH., Desai TJ.,

553 Krasnow MA., Quake SR. 2014. Reconstructing lineage hierarchies of the distal lung

$554 \quad$ epithelium using single-cell RNA-seq. Nature 509:371-375.

555 Tung P-Y., Blischak JD., Hsiao C., Knowles DA., Burnett JE., Pritchard JK., Gilad Y. 2016.

556 Batch effects and the effective design of single-cell gene expression studies. bioRxiv:62919.

557 Usoskin D., Furlan A., Islam S., Abdo H., Lönnerberg P., Lou D., Hjerling-Leffler J.,

558 Haeggström J., Kharchenko O., Kharchenko P V. 2014. Unbiased classification of sensory

559 neuron types by large-scale single-cell RNA sequencing. Nature neuroscience 18:145-153.

560 Yang Z., Zhang H., Yuan Z., Oja E. 2011. Kullback-Leibler divergence for nonnegative matrix

561 factorization. In: Artificial Neural Networks and Machine Learning-ICANN 2011. Springer, $562 \quad 250-257$.

563 Ye X., Liu J., Wu F-X. 2012. Dynamic miRNA-TF-mRNA circuits in mouse lung development.

564 In: 2012 IEEE 6th International Conference on Systems Biology (ISB). IEEE, 256-262.

565 Yuan Z., Oja E. 2005. Projective nonnegative matrix factorization for image compression and 566 feature extraction. In: Image Analysis. Springer, 333-342.

567 Zeisel A., Muñoz-Manchado AB., Codeluppi S., Lönnerberg P., La Manno G., Juréus A., 568 Marques S., Munguba H., He L., Betsholtz C. 2015. Cell types in the mouse cortex and 

hippocampus revealed by single-cell RNA-seq. Science 347:1138-1142.

570

571

572

573

574

575

576

Zhang P., Cao H-Y., Bai L-L., Li W-N., Wang Y., Chen S-Y., Zhang L., Yang L-H., Xu H-T., Wang E-H. 2015. The high expression of TC1 (C8orf4) was correlated with the expression of $\beta$-catenin and cyclin D1 and the progression of squamous cell carcinomas of the tongue. Tumor Biology 36:7061-7067.

Zhu P., Wang Y., Du Y., He L., Huang G., Zhang G., Yan X., Fan Z. 2015. C8orf4 negatively regulates self-renewal of liver cancer stem cells via suppression of NOTCH2 signalling. Nature communications 6.

Biase F., Cao X., Zhong S. 2014. Cell fate inclination within 2-cell and 4-cell mouse embryos revealed by single-cell RNA sequencing. Genome research:gr-177725.

Blake-Palmer KG., Su Y., Smith AN., Karet FE. 2007. Molecular cloning and characterization of a novel form of the human vacuolar H+-ATPase e-subunit: an essential proton pump component. Gene 393:94-100.

Brennecke P., Anders S., Kim JK., Kołodziejczyk AA., Zhang X., Proserpio V., Baying B., Benes V., Teichmann SA., Marioni JC. 2013. Accounting for technical noise in single-cell RNA-seq experiments. Nature methods.

Brunet J-P., Tamayo P., Golub TR., Mesirov JP. 2004. Metagenes and molecular pattern discovery using matrix factorization. Proceedings of the National Academy of Sciences 101:4164-4169.

Brunskill EW., Park J-S., Chung E., Chen F., Magella B., Potter SS. 2014. Single cell dissection of early kidney development: multilineage priming. Development 141:3093-3101. 
590 Buettner F., Natarajan KN., Casale FP., Proserpio V., Scialdone A., Theis FJ., Teichmann SA., 591 Marioni JC., Stegle O. 2015. Computational analysis of cell-to-cell heterogeneity in single592 cell RNA-sequencing data reveals hidden subpopulations of cells. Nature biotechnology $593 \quad 33: 155-160$.

594 Bushati N., Smith J., Briscoe J., Watkins C. 2011. An intuitive graphical visualization technique 595 for the interrogation of transcriptome data. Nucleic acids research 39:7380-9. DOI: $596 \quad 10.1093 / \mathrm{nar} / \mathrm{gkr} 462$.

597 Edgar R., Domrachev M., Lash AE. 2002. Gene Expression Omnibus: NCBI gene expression 598 and hybridization array data repository. Nucleic Acids Research 30:207-210. DOI: $599 \quad 10.1093 /$ nar/30.1.207.

600 Fan J-BJJ-BJ., Salathia N., Liu R., Kaeser GE., Yung YC., Herman JL., Kaper F., Fan J-BJJ-BJ., 601 Zhang K., Chun J., others. 2016. Characterizing transcriptional heterogeneity through 602 pathway and gene set overdispersion analysis. Nature methods.

603 Gao Y., Church G. 2005. Improving molecular cancer class discovery through sparse non604 negative matrix factorization. Bioinformatics 21:3970-3975.

605 Gaujoux R., Seoighe C. 2010. A flexible R package for nonnegative matrix factorization. $B M C$ 606 bioinformatics 11:367.

607 Huang DW., Sherman BT., Lempicki RA. 2008. Systematic and integrative analysis of large 608 gene lists using DAVID bioinformatics resources. Nature protocols 4:44-57.

609 Huang DW., Sherman BT., Lempicki RA. 2009. Bioinformatics enrichment tools: paths toward 610 the comprehensive functional analysis of large gene lists. Nucleic acids research 37:1-13. 
611 Jia Z., Zhang X., Guan N., Bo X., Barnes MR., Luo Z. 2015. Gene Ranking of RNA-Seq Data

612 via Discriminant Non-Negative Matrix Factorization. PloS one 10:e137782.

613 Jiao Y., Widschwendter M., Teschendorff AE. 2014. A systems-level integrative framework for 614 genome-wide DNA methylation and gene expression data identifies differential gene 615 expression modules under epigenetic control. Bioinformatics:btu316.

616 Junker JP., Noël ES., Guryev V., Peterson KA., Shah G., Huisken J., McMahon AP., Berezikov

617 E., Bakkers J., van Oudenaarden A. 2014. Genome-wide RNA Tomography in the

618 Zebrafish Embryo. Cell 159:662-675. DOI: 10.1016/j.cell.2014.09.038.

619 Karolchik D., Barber GP., Casper J., Clawson H., Cline MS., Diekhans M., Dreszer TR., Fujita 620 PA., Guruvadoo L., Haeussler M. 2014. The UCSC genome browser database: 2014 update. $621 \quad$ Nucleic acids research 42:D764-D770.

622 Kharchenko P V., Silberstein L., Scadden DT. 2014. Bayesian approach to single-cell differential 623 expression analysis. Nature methods 11:740-742.

624 Kim D., Pertea G., Trapnell C., Pimentel H., Kelley R., Salzberg SL. 2013. TopHat2: accurate 625 alignment of transcriptomes in the presence of insertions, deletions and gene fusions. $626 \quad$ Genome Biol 14:R36.

627 Kumar RM., Cahan P., Shalek AK., Satija R., DaleyKeyser AJ., Li H., Zhang J., Pardee K.,

628 Gennert D., Trombetta JJ., Ferrante TC., Regev A., Daley GQ., Collins JJ. 2014.

629 Deconstructing transcriptional heterogeneity in pluripotent stem cells. Nature 516:56-61.

$630 \quad$ DOI: $10.1038 /$ nature 13920.

631 Leinonen R., Sugawara H., Shumway M., Collaboration on behalf of the INSD. 2011. The 
633 Liao Y., Smyth GK., Shi W. 2014. featureCounts: an efficient general purpose program for 634 assigning sequence reads to genomic features. Bioinformatics 30:923-930.

635 Love MI., Huber W., Anders S. 2014. Moderated estimation of fold change and dispersion for 636 RNA-Seq data with DESeq2. bioRxiv.

637 Ma X-Y., Wang J-H., Wang J-L., Ma CX., Wang X-C., Liu F-S. 2015a. Malat1 as an 638 evolutionarily conserved lncRNA, plays a positive role in regulating proliferation and 639 maintaining undifferentiated status of early-stage hematopoietic cells. BMC genomics $640 \quad 16: 676$.

641 Ma K., Wang H., Li X., Li T., Su G., Yang P., Wu J. 2015b. Long noncoding RNA MALAT1 642 associates with the malignant status and poor prognosis in glioma. Tumor Biology 36:3355$643 \quad 3359$.

644 van der Maaten L. 2013. Barnes-hut-sne. arXiv preprint arXiv:1301.3342.

645 Van der Maaten L., Hinton G. 2008. Visualizing data using t-SNE. Journal of Machine Learning $646 \quad$ Research 9:85.

647 Macosko EZ., Basu A., Satija R., Nemesh J., Shekhar K., Goldman M., Tirosh I., Bialas AR., 648 Kamitaki N., Martersteck EM. 2015. Highly parallel genome-wide expression profiling of 649 individual cells using nanoliter droplets. Cell 161:1202-1214.

650 McDavid A., Finak G., Chattopadyay PK., Dominguez M., Lamoreaux L., Ma SS., Roederer M., 651 Gottardo R. 2013. Data exploration, quality control and testing in single-cell qPCR-based 652 gene expression experiments. Bioinformatics (Oxford, England) 29:461-7. DOI: 
653 10.1093/bioinformatics/bts714.

654 Monga V., Mihçak MK. 2007. Robust and secure image hashing via non-negative matrix 655 factorizations. Information Forensics and Security, IEEE Transactions on 2:376-390.

656 Moore HC., Johnston M., Nicol SM., Bourdon J-C., Thompson AM., Hutvagner G., Fuller-Pace 657 F V. 2011. An evolutionarily conserved, alternatively spliced, intron in the p68/DDX5 658 DEAD-box RNA helicase gene encodes a novel miRNA. RNA 17:555-562.

659 Pan X. 2014. Single Cell Analysis: From Technology to Biology and Medicine. Single cell 660 biology 3:106. DOI: 10.4172/2168-9431.1000106.

661 Patel AP., Tirosh I., Trombetta JJ., Shalek AK., Gillespie SM., Wakimoto H., Cahill DP., Nahed 662 B V., Curry WT., Martuza RL., Louis DN., Rozenblatt-Rosen O., Suvà ML., Regev A., 663 Bernstein BE. 2014. Single-cell RNA-seq highlights intratumoral heterogeneity in primary 664 glioblastoma. Science 344:1396-1401. DOI: 10.1126/science.1254257.

665 Qi Q., Zhao Y., Li M., Simon R. 2009. Non-negative matrix factorization of gene expression 666 profiles: a plug-in for BRB-ArrayTools. Bioinformatics 25:545-547.

667 Rajapakse M., Tan J., Rajapakse J. 2004. Color channel encoding with NMF for face 668 recognition. In: Image Processing, 2004. ICIP'04. 2004 International Conference on. IEEE, $669 \quad 2007-2010$.

670 Rand WM. 1971. Objective criteria for the evaluation of clustering methods. Journal of the 671 American Statistical association 66:846-850.

672 Reichardt J., Bornholdt S. 2006. Statistical mechanics of community detection. Physical Review 673 E 74:16110. 
674 Robinson MD., McCarthy DJ., Smyth GK. 2010. edgeR: a Bioconductor package for differential 675 expression analysis of digital gene expression data. Bioinformatics 26:139-140.

676 Schlitzer A., Sivakamasundari V., Chen J., Sumatoh HR Bin., Schreuder J., Lum J., Malleret B., 677 Zhang S., Larbi A., Zolezzi F. 2015. Identification of cDC1-and cDC2-committed DC 678 progenitors reveals early lineage priming at the common DC progenitor stage in the bone 679 marrow. Nature immunology 16:718-728.

680 Smaragdis P. 2004. Non-negative matrix factor deconvolution; extraction of multiple sound 681 sources from monophonic inputs. In: Independent Component Analysis and Blind Signal 682 Separation. Springer, 494-499.

683 Tamayo P., Scanfeld D., Ebert BL., Gillette MA., Roberts CWM., Mesirov JP. 2007. Metagene 684 projection for cross-platform, cross-species characterization of global transcriptional states. 685 Proceedings of the National Academy of Sciences 104:5959-5964.

Trapnell C., Cacchiarelli D., Grimsby J., Pokharel P., Li S., Morse M., Lennon NJ., Livak KJ., 687 Mikkelsen TS., Rinn JL. 2014. Pseudo-temporal ordering of individual cells reveals 688 dynamics and regulators of cell fate decisions. Nature biotechnology 32:381.

Treutlein B., Brownfield DG., Wu AR., Neff NF., Mantalas GL., Espinoza FH., Desai TJ., 690 Krasnow MA., Quake SR. 2014. Reconstructing lineage hierarchies of the distal lung 691 epithelium using single-cell RNA-seq. Nature 509:371-375.

692 Tung P-Y., Blischak JD., Hsiao C., Knowles DA., Burnett JE., Pritchard JK., Gilad Y. 2016. 693 Batch effects and the effective design of single-cell gene expression studies. bioRxiv:62919.

694 Usoskin D., Furlan A., Islam S., Abdo H., Lönnerberg P., Lou D., Hjerling-Leffler J., 
695

696

697

698

699

700

701

702

703

704

705

706

707

708

709

710

711

712

713

714

715

716

Haeggström J., Kharchenko O., Kharchenko P V. 2014. Unbiased classification of sensory

neuron types by large-scale single-cell RNA sequencing. Nature neuroscience 18:145-153.

Yang Z., Zhang H., Yuan Z., Oja E. 2011. Kullback-Leibler divergence for nonnegative matrix factorization. In: Artificial Neural Networks and Machine Learning-ICANN 2011. Springer, $250-257$.

Yuan Z., Oja E. 2005. Projective nonnegative matrix factorization for image compression and feature extraction. In: Image Analysis. Springer, 333-342.

Zeisel A., Muñoz-Manchado AB., Codeluppi S., Lönnerberg P., La Manno G., Juréus A., Marques S., Munguba H., He L., Betsholtz C. 2015. Cell types in the mouse cortex and hippocampus revealed by single-cell RNA-seq. Science 347:1138-1142.

\section{Figure legends}

Fig. 1: The workflow of NMFEM. The input can be either FASTQ files or a raw counts table. If FASTQ files are used, they are aligned using TopHat and counted using FeatureCounts (steps shown in brackets). The input or calculated raw counts table are filtered by samples and genes, converted into FPKMs using gene lengths, and normalized by samples. We then run NMF method on them to detect groups of cells, and find the feature genes separating the detected groups. Finally, we feed the feature genes as seed genes in FEM, and generate PPI gene modules that contain highly differentially expressed genes.

Fig. 2: Rand measures comparison of all methods on five datasets. (A) Mouse embryonic lung epithelial E14.5 vs E16.5 (B) HSC vs. MPP1 (C) Glioblastoma MGH29 vs MGH31 (D) Bone marrow dendritic cells (CDP vs MDP) (E) human induced pluripotent stem cell (iPSC) lines with UMI counts. Rand measure ranges from 0 to 1 , where a higher value indicates a 
717 greater clustering accuracy. The error bars show the standard deviation across 30 runs. Results

718 significantly worse than NMF without tSNE by Welch t-test are marked by asterisks. For

719 datasets with more than two groups of cells, the closest pair is selected.

720 Fig. 3: MA-plots of significant or important genes identified by different methods.

721 (A-F) Shown are scRNA-Seq data in the mouse lung distal epithelial cell E14.5 vs. E16.5

722 samples. The blue color highlights the genes selected as "the most significant" by the

723 corresponding methods. $\mathrm{X}$-axis (A-value) is the mean of the gene expression, and $\mathrm{y}$-axis (M-

724 value) is the difference of the gene expression between E16.5 and E14.5 stages.

725 Fig. 4: Network of top 5 modules using the seed genes generated by NMF.

726 Shown are module detection results in the FEM package, using the top 500 most important genes

727 detected by NMF in Fig. 3. scRNA-Seq data in the mouse lung distal epithelial cell E14.5 vs.

728 E16.5 samples are compared, where the red and blue colors indicate up- and down-regulation of

729 genes in E16.5 relative to E14.5, respectively. The top 5 modules are selected by the $p$-values

730 calculated from the internal Monte Carlo method in the FEM package.

731

732 Supporting Information

733 Fig. S1: Errors of labeling in other methods, exemplified with the mouse embryonic lung

734 epithelial dataset. (A) PCA plot showing wrongly clusters samples by K-means. (B-D)

735 Dendrogram showing the clustering results of hierarchical clustering, the red dashed lines are the 736 binary decisions from the roots. 
737 Fig. S2: Overlapping between the feature genes found by the various important gene

738 calling methods in Fig 3. (A) heatmap with each square marking the Jaccard index of the pair.

739 (B) The dendrogram showing the hierarchical clustering results using the distance measured by

740 one minus the Jaccard indices.

741 Fig. S3: The kernel density estimation (KDE) plot showing the frequency of log expression

742 values of "important genes" that separate E14.5 vs. E16.5, as detected by the various methods in

743 Fig. 3.

744 Fig. S4: MA-plots and PPI network modules in other datasets. (A-C) MA-plot highlighting

745 the important genes identified by NMF, on three other datasets: (A) HSC vs. MPP1 (B)

746 Glioblastoma MGH29 vs MGH31 (C) Bone marrow dendritic cells CDP vs. MDP. (D-F)

747 Corresponding top PPI network modules discovered by Spinglass algorithm, using genes

748 identified in (A-C) respectively.

749 Fig. S5: The kernel density estimation (KDE) plot showing distribution of the CV2 of the

750 "important genes" that separate E14.5 vs. E16.5, as detected by the various methods in Fig. 3.

751 Fig. S6: Bar-plot showing the robustness of the top 500 genes selected by NMF. Leave-one-out

752 runs are performed and the selected top 500 genes have over $95 \%$ frequency to be in the top 500

753 gene list, whereas non-top 500 genes have less than $2 \%$ frequency to enter the top 500 gene list.

754 Table S1: FPKM table of the mouse hematopoietic stem cell (HSC) and multipotent progenitor

755 (MPP) cell dataset. 


\section{Figure 1 (on next page)}

\section{The workflow of NMFEM}

The input can be either FASTQ files or a raw counts table. If FASTQ files are used, they are aligned using TopHat and counted using FeatureCounts (steps shown in brackets). The input or calculated raw counts table are filtered by samples and genes, converted into FPKMs using gene lengths, and normalized by samples. We then run NMF method on them to detect groups of cells, and find the feature genes separating the detected groups. Finally, we feed the feature genes as seed genes in FEM, and generate PPI gene modules that contain highly differentially expressed genes. 


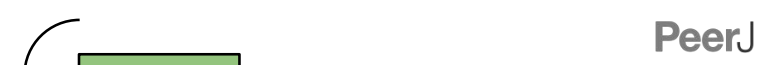

FASTQ

Files

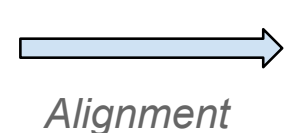

Alignment

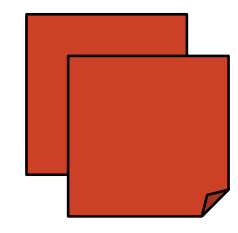

Manuscript to be reviewed

BAM Files

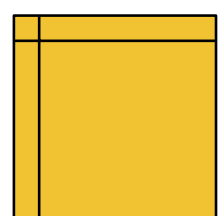

Raw

Counts Table

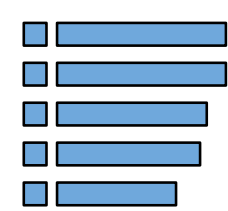

$\prod$

Functional

Modules (FEM)

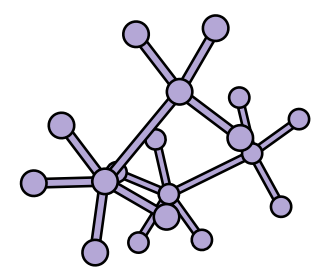

Feature Genes

PPI Modules

\section{NMF}

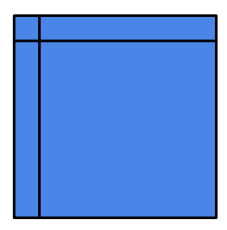

Normalized FPKM

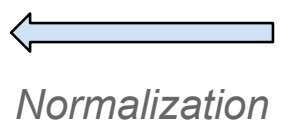

Filtered Counts Table
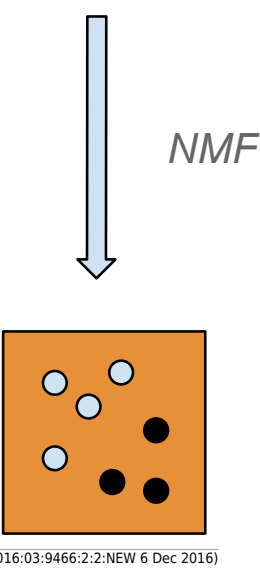

Cell Group

Detection 
Figure 2 (on next page)

Rand measures comparison of all methods on five datasets

(A) Mouse embryonic lung epithelial E14.5 vs E16.5 (B) HSC vs. MPP1 (C) Glioblastoma MGH29 vs MGH31 (D) Bone marrow dendritic cells (CDP vs MDP) (E) human induced pluripotent stem cell (iPSC) lines with UMI counts. Rand measure ranges from 0 to 1 , where a higher value indicates a greater clustering accuracy. The error bars show the standard deviation across 30 runs. Results significantly worse than NMF without tSNE by Welch t-test are marked by asterisks. For datasets with more than two groups of cells, the closest pair is selected. 

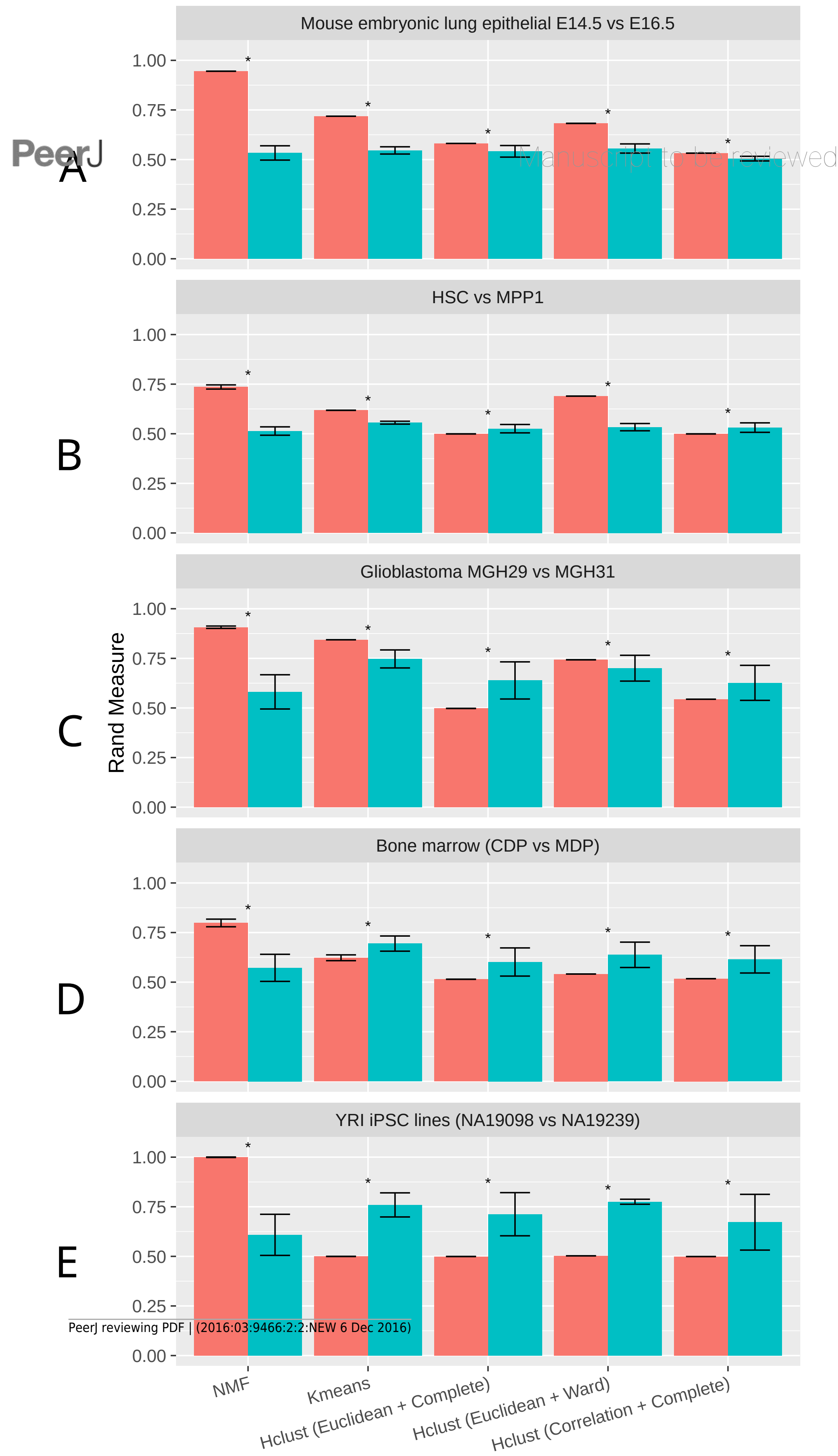

Method 
Figure 3 (on next page)

MA-plots of significant or important genes identified by different methods

Shown are scRNA-Seq data in the mouse lung distal epithelial cell E14.5 vs. E16.5 samples. The blue color highlights the genes selected as "the most significant" by the corresponding methods. X-axis (A-value) is the mean of the gene expression, and $y$-axis (M-value) is the difference of the gene expression between E16.5 and E14.5 stages. 
$A^{e e r J}$

2.5 -

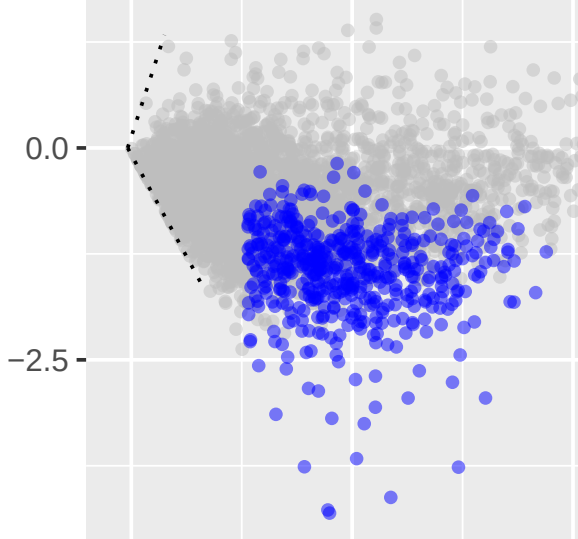

$-5.0-$

$\Sigma$

$-2.5-$

$-5.0-$

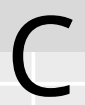

\section{MAST}

$2.5-\square$

DESeq2

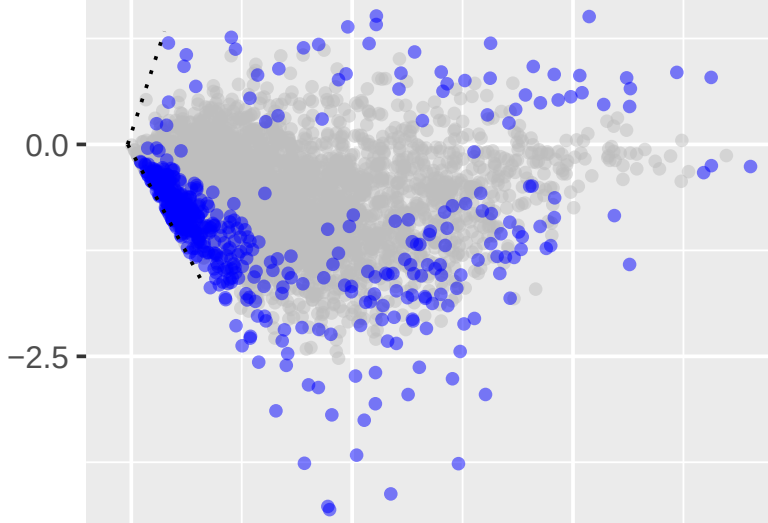

$-5.0-$
B Manuscript to soge reviewed
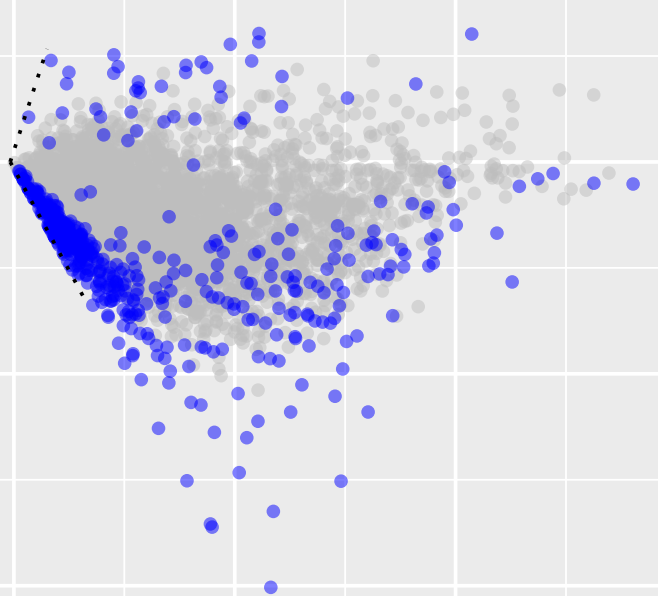

D

Monocle

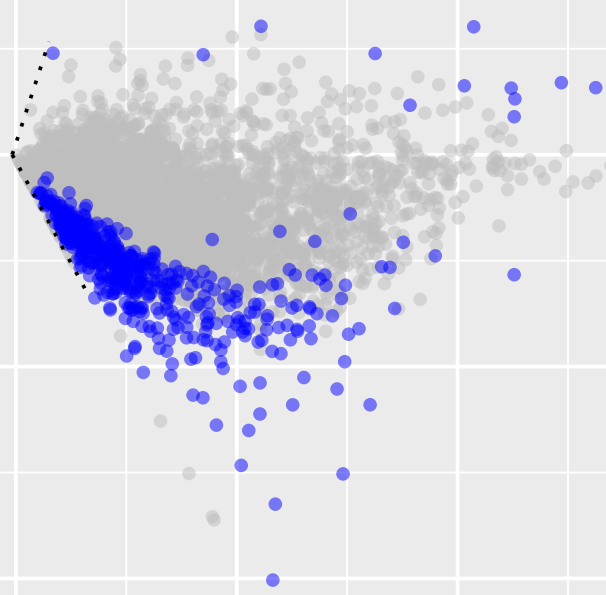

EdgeR

ए

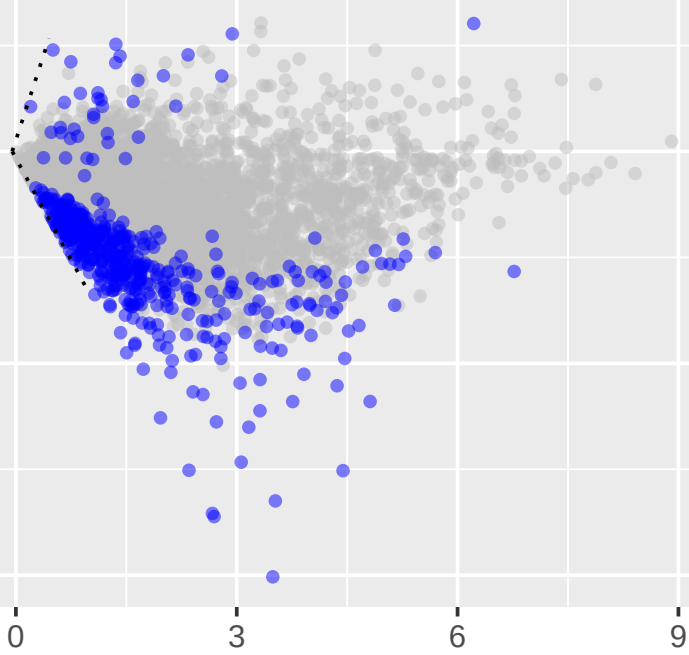


Figure 4 (on next page)

Network of top 5 modules using the seed genes generated by NMF

Shown are module detection results in the FEM package, using the top 500 most important genes detected by NMF in Fig. 3. scRNA-Seq data in the mouse lung distal epithelial cell E14.5 vs. E16.5 samples are compared, where the red and blue colors indicate up- and downregulation of genes in E16.5 relative to E14.5, respectively. The top 5 modules are selected by the $\mathrm{p}$-values calculated from the internal Monte Carlo method in the FEM package. 


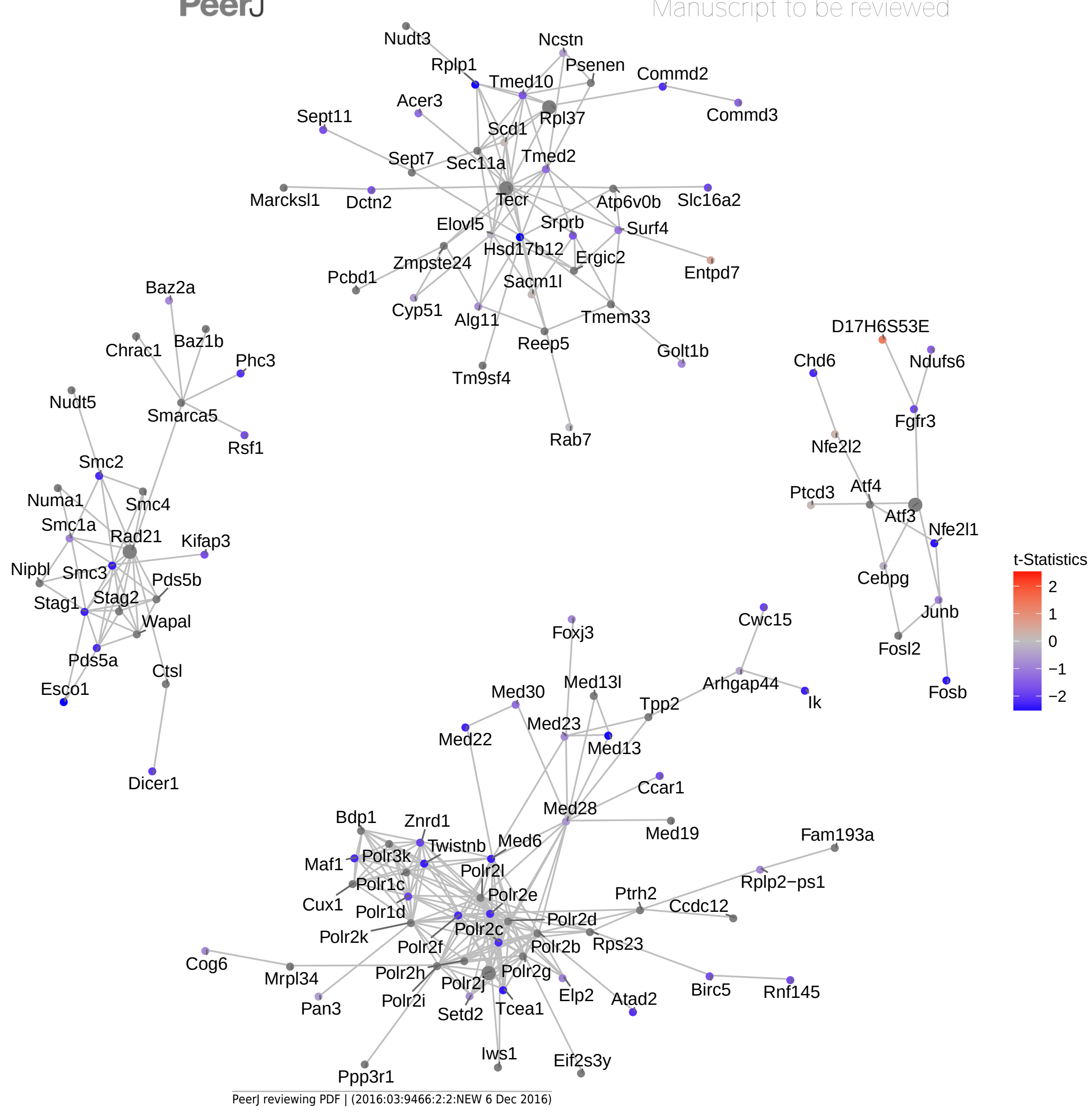

